Cite this: Phys. Chem. Chem. Phys., 2013,

\title{
Infrared multiple photon dissociation (IRMPD) spectroscopy of oxazine dyes
}

15, 5049

Received 24th June 2012, Accepted 6th February 2013

DOI: 10.1039/c3сp00158j

www.rsc.org/pccp

\author{
Robert J. Nieckarz, ${ }^{a}$ Jos Oomens, ${ }^{\text {bcd }}$ Giel Berden, ${ }^{\text {bc }}$ Pavel Sagulenko a and \\ Renato Zenobi*a
}

\section{Introduction}

Over the past ten years, interest in the fluorescent properties of molecular ions in the gas phase has grown substantially. Pioneering investigations by several research groups ${ }^{1-5}$ have shown that some remarkable differences in absorption and emission maxima, quantum yield, and excited state lifetime can exist between a bare fluorophore in the gas-phase and its solvated counterpart. For instance, the fluorescence maximum of gaseous rhodamine $6 \mathrm{G}(\mathrm{R} 6 \mathrm{G})$, a dye not typically described as being sensitive to the polarity of its environment, ${ }^{6}$ was observed to be blue-shifted by $50 \mathrm{~nm}$ with respect to its solvated state. ${ }^{7}$ Fluorescent resonant energy transfer (FRET) has also been successfully demonstrated in the gas-phase, ${ }^{8}$ and more recently, the effect of donor-acceptor distance and ionic charge state of doubly labeled polyproline on FRET efficiency has been investigated. ${ }^{9}$

Gas-phase fluorescence studies give direct insight into the intrinsic spectroscopic properties of an ion. However, most fluorophores do not necessarily retain their fluorescent

\footnotetext{
${ }^{a}$ Department of Chemistry and Applied Biosciences, ETH Zürich, CH-8093 Zürich, Switzerland. E-mail: zenobi@org.chem.ethz.ch; Fax: +41-44-632-1292

${ }^{b}$ FOM Institute for Plasma Physics Rijnhuizen, Edisonbaan 14, 3439MN Nieuwegein, The Netherlands

${ }^{c}$ Radboud University Nijmegen, Institute for Molecules and Materials, FELIX Facility, Toernooiveld 7, 6525ED Nijmegen, The Netherlands. E-mail: joso@science.ru.nl

${ }^{d}$ University of Amsterdam, Science Park 904, 1098XH Amsterdam, The Netherlands
}

properties upon transfer to the gas-phase. The direction and magnitude of shifts in absorption and emission wavelength maxima, as well as in quantum yields, can be quite unpredictable and are often due to conformational changes within the fluorophore upon desolvation and ionization. A definitive structural description of a gaseous fluorophore is essential for the prediction of the associated fluorescent properties.

While the measurement of these fluorescent properties themselves can reveal structural information about an ion, this method of analysis is less than helpful when no fluorescence can be observed. Quantum-chemical calculations of ion structure and energetics can provide insight into the expected gas-phase behavior and help direct future experiments, but it is essential to have experimental data to compare against. Unexpected results, such as the trapping in local minimum structures due to a large kinetic barrier, ${ }^{10-14}$ or the presence of a kinetically inaccessible structure thanks to a catalyzed rearrangement, ${ }^{15}$ can often surface in the dynamic process of ion desolvation. Infrared spectroscopy of mass-selected ions provides a means by which the isomeric-tautomeric composition of a population of gaseous ions can be determined with high confidence. In order to aid gas-phase fluorescent measurements, we investigate here several potential fluorophores using IR multiple photon dissociation (IRMPD) action spectroscopy.

The oxazine family of dyes constitutes a widely used set of fluorescent stains in modern biology and histology. For instance, one notable member, Nile red (NR) is a commonly 
used probe for the investigation of many chemically important systems, and has been studied extensively both experimentally ${ }^{16}$ and theoretically. ${ }^{17}$ Despite its well-characterized fluorescence in solution, NR has eluded all attempts to observe its gas-phase fluorescence.

When multiple protonation sites exist, as is the case for NR, knowledge of the true structure of the gaseous protonated fluorophore is indispensable for interpretation of gas-phase fluorescence data. Vibrational fingerprints of protonated NR, as well as the structurally similar cationic oxazine dyes Nile blue A (NB), Cresyl violet (CV) and Brilliant cresyl blue (BCB), allow for the identification of the protonation site in NR and vibrational band assignments based on experimental data.

\section{Methods}

\subsection{Ion generation}

While NB (Fluka Chemie AG, Switzerland), BCB (Fluka Chemie AG, Switzerland) and CV (Acros Organics, Belgium) were obtained in their protonated form as acetate or perchlorate salts, NR (Acros Organics, Belgium) is sold in its free-base form. Determination of the gas-phase protonation site of NR by electrospray ionization (ESI) is one of the main goals of this study. The dyes were prepared as $\sim 0.5 \mathrm{mM}$ solutions in methanol (99.8\% Acros Organics, Belgium) acidified with about $5 \%$ acetic acid to aid in the protonation of the dye. A Waters Z-Spray source was used as electrospray source (flow rate $10 \mu \mathrm{L} \mathrm{min}{ }^{-1}$, needle potential $2.5-4 \mathrm{kV}$ ), with the entrance cone of the mass spectrometer biased to $50-100 \mathrm{~V}$.

\subsection{IRMPD photodissociation experiments}

IRMPD spectra were recorded at the FELIX facility ${ }^{18}$ in The Netherlands. Only a brief description of the setup will be given since a detailed description has been previously reported. ${ }^{19,20}$ Ions are accumulated in a linear hexapole ion trap before being injected into a Fourier transform ion cyclotron resonance (FTICR) mass spectrometer. Ions of interest were isolated using a waveform generated from the inverse Fourier transform of an ejection window defined by the user in the frequency domain (SWIFT).${ }^{21}$ After isolation, radiation from the free electron laser (wavelength tuned between 900 and $1800 \mathrm{~cm}^{-1}$, pulse energies ranging from $10-30 \mathrm{~mJ}$ ) was introduced into the ICR cell for 3.5-5.3 seconds to induce fragmentation. Subsequently, an excite/detect event was used to collect a transient signal from the FTICR MS. For statistical purposes, this procedure was repeated three times at each irradiation wavelength and the transients from the three measurements were co-added before Fourier transformation to generate a mass spectrum. The extent of fragmentation was quantified by calculating the fragment ion yield,

$$
\text { Yield }=\frac{\sum I_{\text {fragments }}}{I_{\text {parent }}+\sum I_{\text {fragments }}},
$$

where $\sum I_{\text {fragments }}$ represents the integrated intensities of all fragment ions and $I_{\text {parent }}$ represents the intensity of the remaining parent ion within each mass spectrum. This procedure ensured that variability in the initial parent ion number density before irradiation was accounted for. The fragment ion yield was then plotted as a function of laser wave number, with a resolution (step size) of around $\Delta \bar{\nu}=5 \mathrm{~cm}^{-1}$. The frequency was calibrated using a grating spectrometer and the dissociation yield was normalized for laser power fluctuations, assuming linear power dependence, as described previously. ${ }^{22}$

\subsection{Computational details}

To aid in structural and vibrational assignments, electronic structure calculations of the ions were performed using Gaussian 03. ${ }^{23}$ All geometries were optimized and harmonic vibrational frequencies calculated at the B3LYP/6-311+G(d,p) level of theory, a combination that is commonly used to predict the energetics and vibrational frequencies of small ionic species. In order to account for known deficiencies resulting from the use of a simple harmonic oscillator approximation and poor descriptions of long-distance electron correlation, a scaling factor of 1.0102 was used for calculating the relative change in Gibbs free energy of protonation $(\Delta \Delta G)$ for all compounds. In addition, a scaling factor of 0.9679 was applied to all calculated vibrational frequencies ${ }^{24}$ for the generation of simulated IRMPD spectra. For direct comparison to the measured IRMPD spectra, the frequency-scaled calculated vibrational line spectra were convoluted using a Lorentzian line shape function with a full width at half maximum (FWHM) value of $30 \mathrm{~cm}^{-1}$. To gain insight into the thermochemical landscape of the protonation of NR, solution-phase electronic structure calculations using a Polarizable Continuum Model $(\mathrm{PCM})^{25}$ for methanol and the B3LYP/6-311+G(d,p) level of theory were also performed.

In order to quantitatively assess the degree of agreement between the experimental and calculated spectra for various isomers, a correlation parameter, $C$, was defined,

$$
C=\sum_{\bar{\nu}}[\operatorname{IRMPD}(\bar{\nu})-\operatorname{Calc}(\bar{\nu})]^{2}
$$

where $\operatorname{IRMPD}(\bar{\nu})$ is the measured ion fragment yield at a given wave number, $\bar{\nu}$ and $\operatorname{Calc}(\bar{\nu})$ is the intensity from the convoluted spectrum calculated for a particular isomer (where both experimental and theoretical spectra are normalized to one before applying eqn (2)). In standard statistical nomenclature this is referred to as the sum of squared residuals and the smaller this value, the better the agreement between the measurement and the model used to describe the system being investigated.

\section{Results and discussion}

\subsection{Structure assignment}

The Lewis structure and calculated lowest energy structure for each of the oxazine dyes are shown in Fig. 1, where NR (I) is in its neutral state, while the other dyes are protonated at the site as indicated for the salt by the manufacturer. NB (II), CV (III) and BCB (IV) are therefore expected to assume the protonated structures displayed in Fig. 1. Comparison of experimental IRMPD and DFT computed spectra for these dyes can be used 

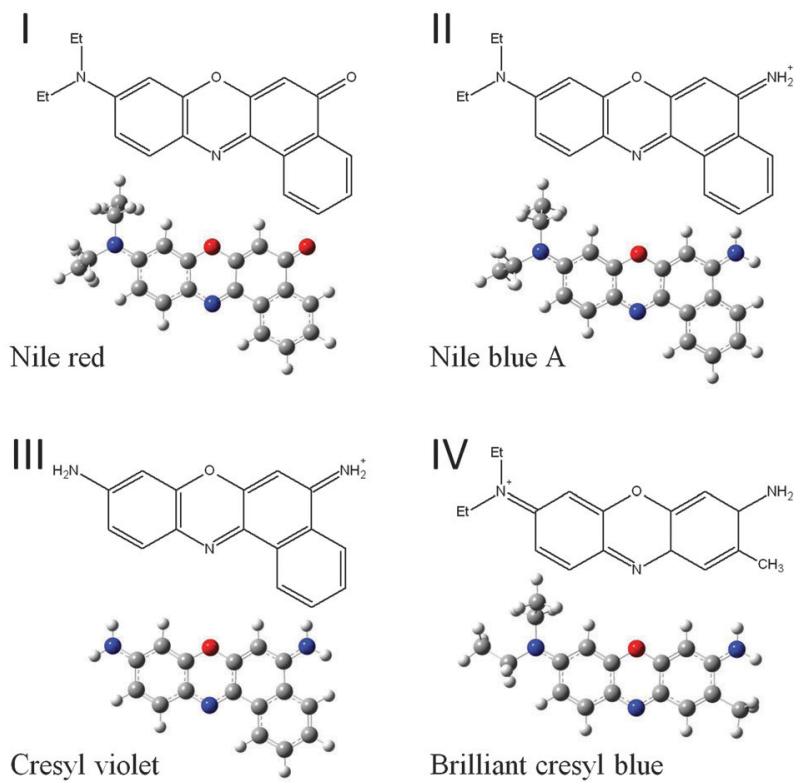

Brilliant cresyl blue

Fig. 1 Lewis and calculated structures of oxazine dyes: (I) Nile red (II) Nile blue A (III) Cresyl violet (IV) Brilliant cresyl blue. Geometries were optimized at the $B 3 L Y P / 6-311+G(d, p)$ level of theory.

as a 'benchmark', verifying our approach to determine the site of protonation in NR. For NR, protonation likely occurs either on the carbonyl oxygen (V), oxazine nitrogen (VI), diethyl amine nitrogen (VII), or oxazine oxygen (VIII), as shown in Fig. 2.
By comparing the experimental IRMPD spectrum of protonated NR to spectra calculated for geometries $\mathbf{V}$ through VIII, the equilibrium gas-phase structure of this oxazine dye is determined below.

3.1.1. Protonated Nile red. The relative energetics of the structures resulting from protonation of NR at each of the heteroatom sites was explored computationally (see Fig. 3). Geometry optimizations were performed both for the isolated (gas-phase) molecule as well as for the molecule in a simulated methanol solution (solution-phase structures not shown) using the PCM method as implemented in Gaussian $03 .{ }^{23}$ While the structure of each of the protonation isomers (tautomers) does not change significantly between the two computational models, the relative Gibbs free energy of protonation, $\Delta \Delta G_{\text {pro }}$, is noticeably affected. In the gas phase, tautomers with the highest degree of charge delocalization are favored. Especially in the carbonyl oxygen protonated form (V), conjugation delocalizes the charge over the carbonyl oxygen and the diethyl amine nitrogen, thus spanning the entire molecule (see Fig. 2). In the next lowest tautomer VI, the oxazine nitrogen becomes conjugated with the naphthalene moiety, delocalizing the charge over this side of the molecule. In contrast, the quaternary amine formed by protonation at the diethyl amine nitrogen in structure VII strongly fixates the charge at this nitrogen atom, while protonation on the oxazine oxygen in VIII tends to fixate the charge at this O-atom.

As seen in Fig. 3, interaction with methanol in solution efficiently delocalizes the charge stabilizing, especially the $\mathbf{v}$<smiles>CCC(CC)c1ccc2c(c1)OC1C=C(C)CC3C(CCCCC13)C2</smiles>

VI

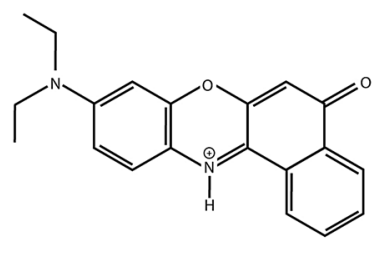

VII<smiles>C=C1CC2Oc3cc(C(CC)CC)ccc3CC3=CC2C1CCC3</smiles>

VIII<smiles>CCC(CC)C1CCC2CC3C(C=C(C)C(C)C4CCCCC34)CC2C1</smiles><smiles></smiles>

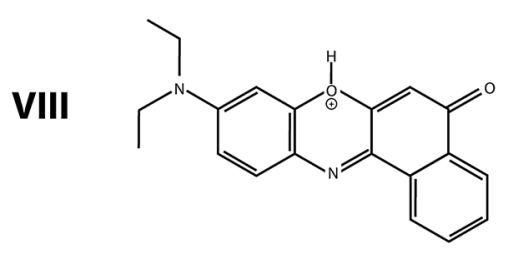

Fig. 2 Resonance structures for various forms of protonated Nile red. Protonation may occur at the (V) carbonyl oxygen, (VI) oxazine nitrogen, (VII) diethyl amine nitrogen, or (VIII) oxazine oxygen. 


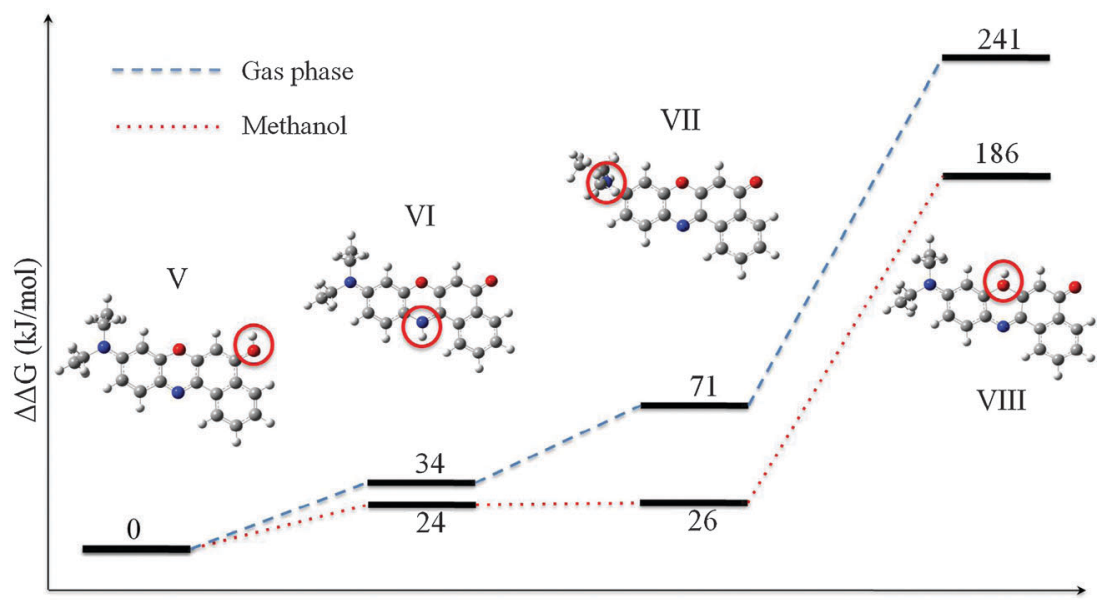

Fig. 3 Calculated relative Gibbs free energy of protonation for four isomers of protonated Nile Red in the gas phase (blue dash) and in methanol solution (red dot).

tautomers with a localized charge in the isolated molecule. In other words, the solvation energy is much higher for diethyl amine-protonated (VII) and oxazine-O protonated (VIII) structures as compared to structures $\mathbf{V}$ and VI, in which the charge is already partially delocalized in their unsolvated form. However, no reordering of the isomers in terms of $\Delta G$ is predicted, but it is interesting to note that solvation makes the diethyl amine protonated isomer nearly as ergonically favorable as the $N$-oxazine protonated form. Despite this significant stabilization effect in solution, it was expected that protonation would take place at the carbonyl oxygen since both solution- and gasphase calculations predicted this form to be at least $24 \mathrm{~kJ} \mathrm{~mol}^{-1}$ lower in energy than any other structure found. Rotational isomers with energies within $10 \mathrm{~kJ} \mathrm{~mol}^{-1}$ of that of the lowest energy structures were found; however, their calculated vibrational frequencies were essentially identical to those of the structures displayed in Fig. 3, and therefore do not influence our search for the site of protonation and hence are not further considered.

Upon irradiation of trapped and thermalized protonated NR at various infrared wavelengths, the primary dissociation channel of the $[\mathrm{M}+\mathrm{H}]^{+}$ion was the loss of 44 Da. This corresponds to the loss of a $\mathrm{C}_{3} \mathrm{H}_{8}$ unit via a 1,2-elimination within the diethyl amine group, which was confirmed by accurate mass data obtained in a collision-induced dissociation (CID) experiment, where the mass of the neutral loss was established as 44.0626 Da (data not shown). These results are consistent with those observed for the low-energy CID of protonated diethyl amine as reported by Reiner et al. ${ }^{26}$ wherein the only alkyl loss observed was that of $\mathrm{C}_{3} \mathrm{H}_{8}$. Since both low-energy CID and IRMPD typically lead to fragmentation via the lowest energy dissociation channel, we conclude that the neutral loss fragment observed in our IRMPD experiments corresponds to $\mathrm{C}_{3} \mathrm{H}_{8}$.

By comparing the calculated vibrational spectra of the various isomers of protonated NR to the experimental IRMPD spectrum, the site of protonation can be identified, which confirms the prediction derived from the thermochemical

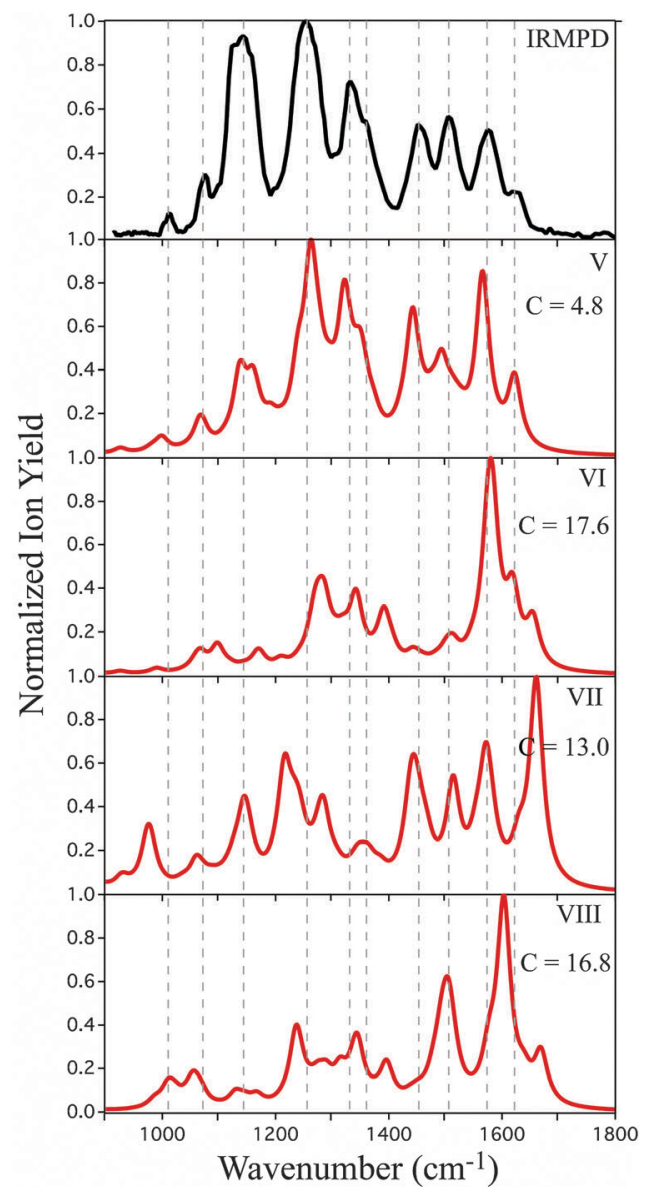

Fig. 4 Experimental IRMPD spectra of protonated Nile red and the four calculated IRMPD spectra corresponding to structures V-VIII (see Fig. 2). All frequencies were scaled by 0.9679 . Dashed lines coincide with the peaks of the IRMPD spectrum and are meant to guide the eye. The calculated correlation parameter is indicated for each isomer.

calculations. As seen in Fig. 4, all vibrational bands and features observed in the measured IRMPD spectrum match the features seen in the spectrum calculated for $\mathbf{V}$, while 
significant deviations are observed between the IRMPD spectrum and the spectra calculated for VI-VIII. This observation is supported by inspection of the correlation parameter $C$, which is smallest for the spectrum predicted for the thermodynamically favored isomer $\mathbf{V}$.

The high degree to which the IRMPD spectrum matches the calculated spectrum for $\mathbf{V}$ gives us confidence that the method and basis set chosen for this study were appropriate. For the most intense bands identified in this spectrum, vibrational mode assignments are given in Table 1 . Of these bands, the $\mathrm{CH}_{3}$ rock at $1075 \mathrm{~cm}^{-1}$ and the coupled $\mathrm{C}-\mathrm{C}$ and $\mathrm{C}-\mathrm{H}$ bending modes of the rings around $1100-1350 \mathrm{~cm}^{-1}$ act as useful diagnostic bands and a point of comparison to the other oxazine dye systems studied (discussed below in Sections 3.1.2 and 3.2).

3.1.2. Nile blue $A$, Cresyl violet and Brilliant cresyl blue. Since NB, CV and BCB dyes were obtained as salts, the protonation site was assumed to be that indicated by the manufacturer, which corresponds to the lowest energy structure in all cases. Despite this, several interesting observations can be made based on the measured IRMPD spectra, accessible fragmentation pathways, as well as the general agreement between the experimentally recorded spectra and the calculated counterparts. The only isomers that were expected to exist were the rotational isomers associated with the amine or diethyl-amine moieties. Similar to protonated NR, calculated spectra for such conformers did not provide evidence that their presence inside the FTICR would alter the recorded IRMPD spectrum (i.e. no significant changes in the calculated vibrational band positions). As seen in Fig. 5, the vibrational bands observed in the recorded IRMPD spectra and those predicted by convolution of the calculated line spectra $\left(\mathrm{FWHM}=30 \mathrm{~cm}^{-1}\right)$ are in reasonably good agreement. The most intense vibrational bands identified for these compounds are also given in Table 1.

Some deviations between experimental and calculated spectra appear to be observed for Cresyl violet and Brilliant cresyl blue, particularly in the low intensities observed at frequencies above $1400 \mathrm{~cm}^{-1}$. The calculated correlation parameters are indeed somewhat higher than for the other systems. We therefore investigate $\mathrm{CV}$ in more detail here by comparing its experimental spectrum to spectra calculated for all four conceivable protonation tautomers (see Fig. 6). However, in terms of band positions, we conclude that the spectra calculated for structures protonated at the oxazine nitrogen $(\boldsymbol{b})$, amine nitrogen $(\boldsymbol{c})$ and oxazine oxygen $(\boldsymbol{d})$ do not provide a better match than that for the structure protonated at the imine nitrogen $(\boldsymbol{a})$. Moreover, these alternative structures $(\boldsymbol{b}-\boldsymbol{d})$ are all more than $100 \mathrm{~kJ} \mathrm{~mol}^{-1}$ higher in energy, which is due to their lower degree of charge delocalization, as was also found

Table 1 Description of the vibrational bands observed in IRMPD spectra for protonated Nile red, Nile blue A, Cresyl violet, and Brilliant cresyl blue

\begin{tabular}{|c|c|c|c|c|}
\hline Description of vibrational band & Protonated Nile red & Nile blue A & Cresyl violet & Brilliant cresyl blue \\
\hline (Ring breathing $+\mathrm{CH}$ rock $/ \mathrm{NH}_{2}$ rock) or $\left(\mathrm{CH}_{3}\right.$ rock) & 1010 & 1010 & 999 & 1007 \\
\hline $\mathrm{CH}_{3}$ rock in diethyl amine & 1075 & 1075 & - & 1075 \\
\hline $\mathrm{CH}$ bending in rings $+\mathrm{OH} / \mathrm{NH}_{2}$ rock & 1122 & 1122 & 1122 & 1142 \\
\hline $\mathrm{CH}$ bending in rings & 1158 & 1158 & - & - \\
\hline $\mathrm{CH}$ bending & 1254 & 1260 & 1250 & 1243 \\
\hline Ring and $\mathrm{CH}$ bending & - & - & 1281 & 1273 \\
\hline Ring and $\mathrm{CH}$ bending & - & - & - & 1311 \\
\hline $\mathrm{CH}$ bending in rings + diethyl amine $\mathrm{CH}$ bend/rock & 1332 & 1328 & - & 1368 \\
\hline $\mathrm{CH}_{3}$ scissor + various ring stretches & 1451 & 1451 & 1462 & 1456 \\
\hline Asymm. ring stretches & 1504 & 1504 & - & - \\
\hline $\mathrm{NH}_{2}$ scissor + asymm. stretch of $\mathrm{C}$ rings & 1574 & 1574 & 1550 & 1590 \\
\hline $\mathrm{NH}_{2}$ scissor + symm. stretch of diethyl-containing ring & 1624 & 1631 & 1631 & 1624 \\
\hline
\end{tabular}

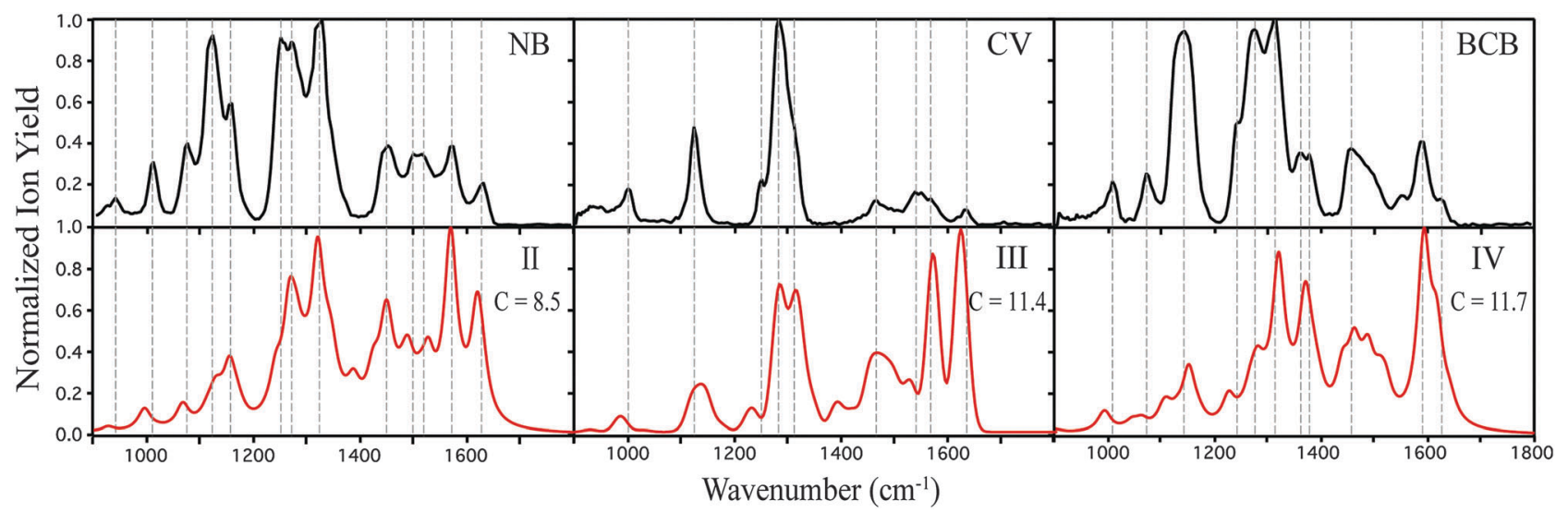

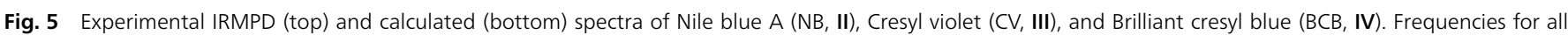
species are scaled by the same factor, 0.9679. Dashed lines help to guide the eye. The calculated correlation parameter is indicated for each isomer. 

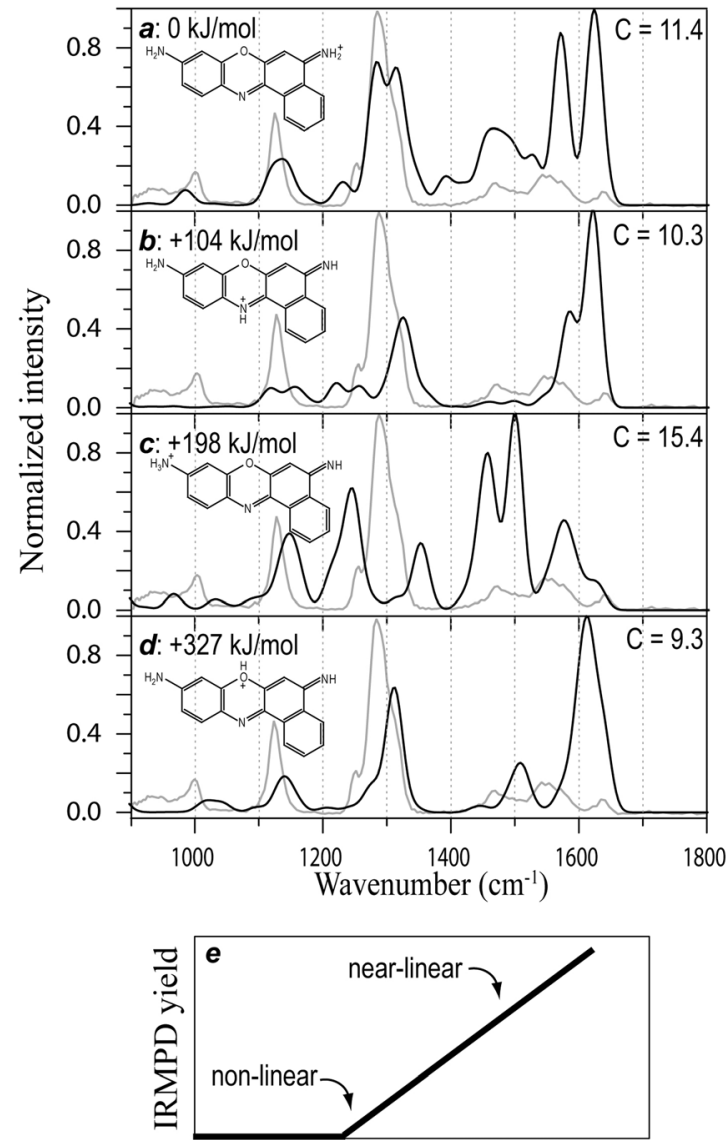

Pulse energy (arb.u.)

Fig. 6 Calculated IR spectra for 4 different protonation sites of Cresyl Violet compared to the experimental spectrum (gray in panels a-d). Panel e shows the typical dependence of the IRMPD signal on laser pulse energy; it is seen that a linear power correction is not valid close to the threshold.

for Nile Red. We therefore conclude that structure $\boldsymbol{a}$ must be the correct one and that the calculated correlation parameter $C$ does not provide a good guide in this case. It appears though that the anomalous values of $C$ are induced by deviations in relative band intensities rather than by deviations in band positions. Below, we argue that the intensity deviations are introduced by the experimental method used.

As for protonated NR, the main dissociation channel observed for the diethyl-amine containing compounds NB and $\mathrm{BCB}$ was the loss of $\mathrm{C}_{3} \mathrm{H}_{8}$ via a 1,2-elimination within the diethyl-amine moiety. $\mathrm{CV}$ on the other hand does not contain a diethyl-amine moiety (Fig. 1) and exhibits loss of $\mathrm{NH}_{3}$ as its lowest energy dissociation pathway. Experimental insight into the relative energetics of the fragmentation pathways for these oxazine dyes was obtained from a comparison of the IRMPD ion yields observed for the set of dye compounds. In the case of CV, longer irradiation times $(5.3 \mathrm{~s})$ were required to generate the IRMPD spectrum shown in Fig. 5. Although all ion yields in Fig. 5 have been normalized to 1 , the absolute yield was much lower for CV than for the other dyes, indicating a higher energy fragmentation for this species. We suspect that this causes the larger discrepancies between the calculated and experimental spectrum for CV. The higher barrier to dissociation is suspected to increase non-linearities in the IR induced dissociation efficiency, arising from the threshold below which no dissociation is observed ${ }^{27-29}$ (the typical behaviour is sketched in Fig. 6e). Towards higher frequencies, where the pulse energies of FELIX gradually decrease, the linear power correction applied to the IRMPD yield is no longer adequate (see Fig. 6e) and vibrational bands therefore appear attenuated.

Finally, a general mismatch between calculated and measured intensities is observed for the feature near $1150 \mathrm{~cm}^{-1}$ for NR, NB and BCB. We suspect that some saturation occurs (due to strong depletion of the ion cloud) on the strongest features in the spectra between 1200 and $1400 \mathrm{~cm}^{-1}$, so that the $1150 \mathrm{~cm}^{-1}$ feature appears too intense in the experimental spectra.

It is also of interest to compare the experimental spectra of this family of structurally related dyes among each other, which can be used to confirm the assignments made based upon the comparison with calculated spectra. The benefit of this type of analysis is that no speculation related to the use of frequency scale factors is necessary. Fig. 7 presents a comparison of the experimental IRMPD spectra collected for the four oxazine dyes, where the red dashed lines are meant to help guide the eye. Descriptions of the observed vibrational bands are given in Table 1.

IRMPD spectra collected for this series of structurally related dyes are dominated by delocalized normal modes pertaining to various ring and substituent vibrations, but show many

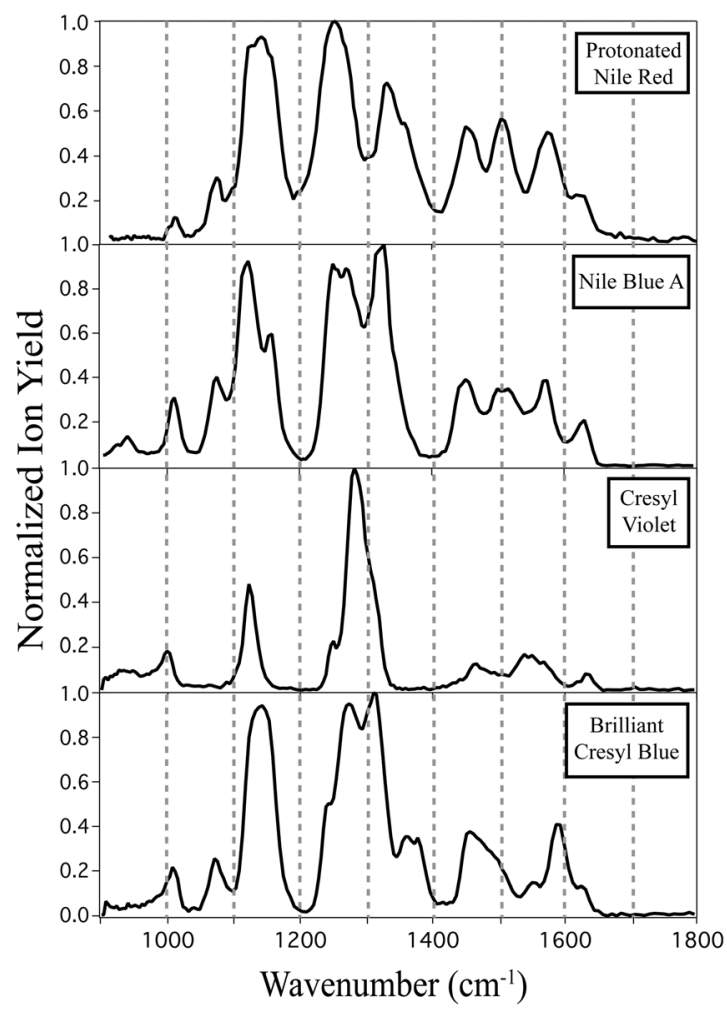

Fig. 7 Comparison of measured IRMPD spectra for structurally related oxazine dyes. 
important differences which are useful for structural assignment. In the case of $\mathrm{CV}$, which contains no diethyl amine moiety, the lack of a band at $1075 \mathrm{~cm}^{-1}$ that is clearly visible in the other three spectra turns out to be a useful diagnostic. Thus, the bands close to $1075 \mathrm{~cm}^{-1}$ are assigned as $\mathrm{CH}_{3}$ rocking modes of the diethyl amine moiety, in agreement with the vibrational assignments suggested by the calculated spectra for these systems. In addition, the spectrum of CV does not exhibit any features in the $1300-1400 \mathrm{~cm}^{-1}$ region, which can also be attributed to the diethyl amine moiety found in the other three structures. Upon inspection of the calculated spectra, it is confirmed that the bands observed in the spectra of protonated NR, NB and BCB between 1325 and $1370 \mathrm{~cm}^{-1}$ arise from a coupling of various in-plane $\mathrm{CH}$ bends of rings with the $\mathrm{CH}$ bends/rock of the diethyl amine moiety. For this reason, NB and protonated NR produce similar vibrational activity in this region while $\mathrm{BCB}$, the only phenoxazine in the series, exhibits a band that is blue shifted by about $40 \mathrm{~cm}^{-1}$. From a close comparison of experiment to calculation, a mixed-character band including various $\mathrm{CH}$ bending and $\mathrm{OH}$ or $\mathrm{NH}_{2}$ rocking motions was identified at $1122 \mathrm{~cm}^{-1}$. Again, the different ring structure of BCB induces a blue shift of about $20 \mathrm{~cm}^{-1}$.

\section{Conclusion}

IRMPD spectra for cationic Nile blue A, Cresyl violet, Brilliant cresyl blue and protonated Nile red in the range-of 900 to $1800 \mathrm{~cm}^{-1}$ were recorded. From a comparison of the measured IRMPD spectrum to various calculated spectra, the site of protonation of Nile red was confidently determined to be the carbonyl oxygen, in agreement with thermochemical calculations. Band assignments of several features in the spectrum are consistent with a comparison with the experimental spectra of structurally similar dyes, as well as with the calculated spectra. Although it is now clear that ionization of NR via electrospray results in the protonated form, $\mathbf{V}$, further experimental and theoretical study will be required to determine if the loss of fluorescence of NR upon transfer to the gas phase is due to desolvation or to the addition of charge through protonation. Knowledge of the gas-phase conformation of protonated NR will simplify calculation of the adiabatic electronic excitation energies and allow for a meaningful computational investigation of this system.

\section{Acknowledgements}

The skillful support by Dr Britta Redlich and others at the FELIX staff is gratefully acknowledged. This work is part of the research program of FOM, which is financially supported by NWO. JO gratefully acknowledges support from the Stichting Physica.

\section{References}

1 A. T. Iavarone and J. H. Parks, J. Am. Chem. Soc., 2005, 127, 8606-8607.
2 B. Cage, J. Friedrich, R. B. Little, Y. S. Wang, M. A. McFarland, C. L. Hendrickson, N. Dalal and A. G. Marshall, Chem. Phys. Lett., 2004, 394, 188-193.

3 Q. Bian, M. W. Forbes, F. O. Talbot and R. A. Jockusch, Phys. Chem. Chem. Phys., 2010, 12, 2590-2598.

4 V. Frankevich, X. Guan, M. Dashtiev and R. Zenobi, Eur. J. Mass Spectrom., 2005, 11, 475-482.

5 Y. Wang, C. L. Hendrickson and A. G. Marshall, Chem. Phys. Lett., 2001, 334, 69-75.

6 D. A. Hinckley, P. G. Seybold and D. P. Borris, Spectrochim. Acta, 1986, 42, 747-754.

7 K. Chingin, V. Frankevich, R. M. Balabin, K. Barylyuk, H. W. Chen, R. Wang and R. Zenobi, Angew. Chem., Int. Ed., 2010, 49, 2358-2361.

8 M. Dashtiev, V. Azov, V. Frankevich, L. Scharfenberg and R. Zenobi, J. Am. Soc. Mass Spectrom., 2005, 16, 1481-1487.

9 F. O. Talbot, A. Rullo, H. Yao and R. A. Jockusch, J. Am. Chem. Soc., 2010, 132, 16156-16164.

10 T. Rocha-Rinza, O. Christiansen, J. Rajput, A. Gopolan, D. B. Rahbek, L. H. Andersen, A. V. Bochenkova, A. A. Granovsky, K. B. Bravaya, A. V. Nemukhin, K. L. Christiansen and M. B. Nielsen, J. Phys. Chem. A, 2009, 113, 9442-9449.

11 Z. Tian and S. R. Kass, Angew. Chem., Int. Ed., 2009, 48, 1321-1323.

12 J. D. Steill and J. Oomens, J. Am. Chem. Soc., 2009, 131, 13570-13571.

13 T. M. Chang, J. S. Prell, E. R. Warrick and E. R. Williams, J. Am. Chem. Soc., 2012, 134, 15805-15813.

14 M. Almasian, J. Grzetic, J. van Maurik, J. D. Steill, G. Berden, S. Ingemann, W. J. Buma and J. Oomens, J. Phys. Chem. Lett., 2012, 3, 2259-2263.

15 R. A. Marta, R. H. Wu, K. R. Eldridge, J. K. Martens and T. B. McMahon, Phys. Chem. Chem. Phys., 2010, 12, 3431-3442.

16 A. K. Dutta, K. Kamada and K. Ohta, J. Photochem. Photobiol., A, 1996, 93, 57-64.

17 P. O. Tuck, R. C. Mawhinney and M. Rappon, Phys. Chem. Chem. Phys., 2009, 11, 4471-4480.

18 D. Oepts, A. F. G. Van der Meer and P. W. Van Amersfoort, Infrared Phys. Technol., 1995, 36, 297-308.

19 J. J. Valle, J. R. Eyler, J. Oomens, D. T. Moore, A. F. G. van der Meer, G. von Helden, G. Meijer, C. L. Hendrickson, A. G. Marshall and G. T. Blakney, Rev. Sci. Instrum., 2005, 76, 023103.

20 D. W. Zhao, J. Langer, J. Oomens and O. Dopfer, J. Chem. Phys., 2009, 131, 184307.

21 A. G. Marshall, T.-C. L. Wang and T. L. Ricca, J. Am. Chem. Soc., 1985, 107, 7893-7897.

22 A. Lagutschenkov, J. Langer, G. Berden, J. Oomens and O. Dopfer, Phys. Chem. Chem. Phys., 2011, 13, 2815-2823.

23 M. J. Frisch, G. W. Trucks, H. B. Schlegel, G. E. Scuseria, M. A. Robb, J. R. Cheeseman, J. A. Montgomery, T. Vreven, K. N. Kudin, J. C. Burant, J. M. Millam, S. S. Iyengar, J. Tomasi, V. Barone, B. Mennucci, M. Cossi, G. Scalmani, N. Rega, G. A. Petersson, H. Nakatsuji, M. Hada, M. Ehara, K. Toyota, R. Fukuda, J. Hasegawa, M. Ishida, T. Nakajima, 
Y. Honda, O. Kitao, H. Nakai, M. Klene, X. Li, J. E. Knox, H. P. Hratchian, J. B. Cross, V. Bakken, C. Adamo, J. Jaramillo, R. Gomperts, R. E. Stratmann, O. Yazyev, A. J. Austin, R. Cammi, C. Pomelli, J. W. Ochterski, P. Y. Ayala, K. Morokuma, G. A. Voth, P. Salvador, J. J. Dannenberg, V. G. Zakrzewski, S. Dapprich, A. D. Daniels, M. C. Strain, O. Farkas, D. K. Malick, A. D. Rabuck, K. Raghavachari, J. B. Foresman, J. V. Ortiz, Q. Cui, A. G. Baboul, S. Clifford, J. Cioslowski, B. B. Stefanov, G. Liu, A. Liashenko, P. Piskorz, I. Komaromi, R. L. Martin, D. J. Fox, T. Keith, A. Laham, C. Y. Peng, A. Nanayakkara, M. Challacombe, P. M. W. Gill, B. Johnson, W. Chen, M. W. Wong, C. Gonzalez and
J. A. Pople, Gaussian 03, Revision D.01, Gaussian Inc., Wallingford CT, 2004.

24 M. P. Andersson and P. Uvdal, J. Phys. Chem. A, 2005, 109, 2937-2941.

25 M. Cossi, G. Scalmani, N. Rega and V. Barone, J. Chem. Phys., 2002, 117, 43-54.

26 E. J. Reiner, A. Harrison and R. D. Bowen, Can. J. Chem., 1989, 67, 2081-2088.

27 L. MacAleese and P. Maitre, Mass Spectrom. Rev., 2007, 26, 583-605.

28 A. Simon, C. Joblin, N. Polfer and J. Oomens, J. Phys. Chem. A, 2008, 112, 8551-8560.

29 F. Calvo and P. Parneix, ChemPhysChem, 2012, 13, 212-220. 